\title{
A Fractional-Order Sequential Hybrid System with an Application to a Biological System
}

\author{
Hasib Khan, ${ }^{1}$ Hashim M. Alshehri, ${ }^{2}$ and Zareen A. Khan ${ }^{3}{ }^{3}$ \\ ${ }^{1}$ Department of Mathematics, Shaheed Benazir Bhutto University Sheringal Dir Upper, Sheringal, \\ Khyber Pakhtunkhwa, Pakistan \\ ${ }^{2}$ Mathematics Department, Faculty of Sciences, King Abdulaziz University, Jeddah 21521, Saudi Arabia \\ ${ }^{3}$ Department of Mathematical Sciences, College of Science, Princess Nourah bint Abdulrahman University, Riyadh, Saudi Arabia
}

Correspondence should be addressed to Zareen A. Khan; zakhan@pnu.edu.sa

Received 7 May 2021; Accepted 14 July 2021; Published 23 July 2021

Academic Editor: Constantin Udriste

Copyright (c) 2021 Hasib Khan et al. This is an open access article distributed under the Creative Commons Attribution License, which permits unrestricted use, distribution, and reproduction in any medium, provided the original work is properly cited.

\begin{abstract}
With the help of Banach's fixed-point approach and the Leray-Schauder alternative theorem, we produced existence results for a general class of fractional differential equations in this paper. The proposed problem is more comprehensive and applicable to real-life situations. As an example of how our problem might be used, we have created a fractional-order COVID-19 model whose solution is guaranteed by our results. We employed a numerical approach to solve the COVID-19 model, and the results were compared for different fractional orders. Our numerical results for fractional orders follow the same pattern as the classical example of order 1 , indicating that our numerical scheme is accurate.
\end{abstract}

\section{Introduction}

In science and engineering, fractional-order operators have lately been investigated for the modeling of dynamical systems. There are operators based on singular kernels and nonsingular kernels. It is tough to determine which operator is the best at the moment, but researchers are constantly analyzing various operators for new features and uses. We have seen that in the vast majority of cases, researchers must compare their findings to the traditional results in terms of accuracy, stability, and simulations. Atangana and Araz focused on the modeling and existence results of the COVID-19 model $[1,2]$. The area of fractional calculus is still open for the researchers to investigate nonlinear models for their theoretical and computational studies with the help of [6-9].

In order to highlight the literature for the existence results and numerical simulations and their applications, we present some examples. Recently, Ahmad et al. [3] discussed a fractional-order COVID-19 model for the existence, uniqueness, and comparative analysis with the existing integer-order model. Babakhani and Daftardar-Gejji [4] studied a boundary value problem of fractional order for the existence of results and presented some applications of their results. Tuan et al. [5] gave some theoretical and computational studies of a fractional-order COVID-19 model for the existence and numerical simulations by the help of Haar wavelets approach. Zhang et al. [10] investigated an impulsive integrodifferential equation for the existence of results and applications.

Boundary value problems (BVPs) with lower-order fractional derivatives and either constant or linear boundary conditions are considered in the majority of these papers. However, there are many cases where nonlinear circumstances at the boundary and differential equations are possible. For example, in case of head flow problems, there are possibilities to have some source or sink on both sides of the boundary (at $x=0$ and $x=1$ ) which may be nonlinear functions and a controller at $x=\zeta_{0}\left(0<\zeta_{0}<1\right)$. Okuonghae and Omame [11] studied a nonlinear system of hybrid fractional differential equations (FDEs) for the existence and applications of solutions. The purpose of this paper is to 
investigate existence results for BVPs involving nonlinear boundary conditions at both ends; that is, we study the following class of two-point BVPs:

$$
{ }^{c} \mathscr{D}^{\vartheta_{i}}\left[{ }^{c} \mathscr{D}^{\omega_{i}^{* *}} u_{i}^{*}(t)-\sum_{1}^{m} h_{i}\left(t, u_{i}^{*}(t)\right)\right]=-f_{i}\left(t, u_{i}^{*}(t)\right), \quad t \in I=[0,1], u_{i}^{*}(0)=0, u_{i}^{*}(1)=0
$$

where the fractional orders $0<\vartheta_{i} \leq 1$ and $0 \leq \omega_{i}^{* *} \leq 1$, the functions $u_{i}^{*}: \mathrm{I} \longrightarrow \mathscr{R}_{e}$ are fractional-order differentiable functions for $i=1,2, \ldots, m$, and $f_{i}: \mathrm{I} \times \mathscr{R}_{e} \longrightarrow$ $\mathscr{R}_{e}-\{0\}$ and $h_{i}: \mathrm{I} \times \mathscr{R}_{e} \longrightarrow \mathscr{R}_{e}(i=1,2, \ldots, m)$ satisfy the Caratheodory conditions. The fractional-order derivatives ${ }^{c} \mathscr{D}^{9} c \mathscr{D}^{\omega_{i}^{* *}}$ are in Caputo's sense. To the best of our knowledge, existence, uniqueness, and stability results had never been studied for BVP (1). Such situation may have importance in application point of view and also in theoretical development and can be studied in the work of Dhage in $[12-14]$ and the reference therein.

\section{Existence Criteria}

Lemma 1. For integrable functions $f_{i}$ and $h_{i}$ on $I$, problem (1) has integral representation given by

$$
u_{i}^{*}(t)=\int_{0}^{1} K_{\omega_{i}, \vartheta_{i}}(s, t) f_{i}\left(s, u_{i}^{*}(s)\right) \mathrm{d} s,
$$

where $K_{\omega_{i}}(s, t)$ is Green's function given by

$$
K_{\omega_{i}, \vartheta_{i}}(s, t)=\frac{1}{\Gamma\left(\omega_{i}^{* *}+\vartheta_{i}\right)} \begin{cases}(1-s)^{\omega_{i}^{* *}+\vartheta_{i}-1} ; & t \leq s, \\ (1-s)^{\omega_{i}^{* *}+\vartheta_{i}-1}-(t-s)^{\omega_{i}^{* *}+\vartheta_{i}-1}, & s \leq t,\end{cases}
$$

for $i=1,2, \ldots, m$.

Proof. Applying integral $\left(I^{9_{i}}\right)$ to problem (1), we obtain

$$
{ }^{c} \mathscr{D}^{\omega_{i}^{* *}} u_{i}^{*}(t)-\sum_{1}^{m} I^{\omega_{i}} h_{i}\left(t, u_{i}^{*}\left(\delta_{i}\right)\right)=-I^{\vartheta_{i}} f_{i}\left(t, u_{i}^{*}(t)\right)+C_{1},
$$

for $i=1,2, \ldots, m$. By the help of $u_{i}^{*}(0)=0$, we have $C_{1}=0$ and hence, we obtain

$$
{ }^{c} \mathscr{D}^{\omega_{i}^{* *}} u_{i}^{*}(t)=\sum_{1}^{m} h_{i}\left(t, u_{i}^{*}\left(\delta_{i}\right)\right)-I^{\vartheta_{i}} f_{i}\left(t, u_{i}^{*}(t)\right)
$$

$$
\begin{aligned}
& \text { Now, applying } I^{\vartheta_{i}}, \text { for } i=1,2, \ldots, m \text { on }(5), \text { we have } \\
& u_{i}^{*}(t)=\sum_{1}^{m} \mathscr{J}^{\omega_{i}^{* *}} h_{i}\left(t, u_{i}^{*}\left(\delta_{i}\right)\right)-I^{\vartheta_{i}+\omega_{i}^{* *}} f_{i}\left(t, u_{i}^{*}(t)\right)+K_{1} .
\end{aligned}
$$

By the use of initial condition $u_{i}^{*}(1)=0$, for $i=1,2, \ldots, m$ and (6), we have $K_{1}=\left.I^{\vartheta_{i}+\omega_{i}^{* *}} f_{i}\left(t, u_{i}^{*}(t)\right)\right|_{t=1}$. Ultimately, we have the following solution:

$$
\begin{aligned}
u_{i}^{*}(t) & =\left.I^{\vartheta_{i}+\omega_{i}^{* *}} f_{i}\left(t, u_{i}^{*}(t)\right)\right|_{t=1}-I^{\vartheta_{i}+\omega_{i}^{* *}} f_{i}\left(t, u_{i}^{*}(t)\right) \\
& =\frac{1}{\Gamma\left(\vartheta_{i}+\omega_{i}^{* *}\right)} \int_{0}^{1}(1-s)^{\vartheta_{i}+\omega_{i}^{* *}-1} f_{i}\left(s, u_{i}^{*}(s)\right) \mathrm{d} s-\frac{1}{\Gamma\left(\vartheta_{i}+\omega_{i}^{* *}\right)} \int_{0}^{t}(t-s)^{\vartheta_{i}+\omega_{i}^{* *}-1} f_{i}\left(s, u_{i}^{*}(s)\right) \mathrm{d} s \\
& =\int_{0}^{1} K_{\omega_{i}, \vartheta_{i}}(t, s) f_{i}\left(s, u_{i}^{*}(s)\right) \mathrm{d} s .
\end{aligned}
$$

$K_{\omega, 9_{i}}(s, t)$ are Green's functions defined in (3), for $i=1,2, \ldots, m$.
Here, we introduce a Banach's space $\mathscr{B}=\left\{f_{i}(t)\right.$ : $f_{i}(t) \in \mathscr{C}([0,1], \mathbb{R})$, for $\left.t \in[0,1]\right\}$, with a norm 
$\left\|f_{i}\right\|=\max _{t \in[0,1]} f_{i}(t)$, for $i=1,2, \ldots, m$. Let us a define an operator $\quad \mathscr{T}_{i}: \mathscr{C}([0,1], \mathbb{R}) \longrightarrow \mathscr{C}([0,1], \mathbb{R}), \quad$ for $\quad i=$ $1,2, \ldots, m$, such that

$$
\begin{aligned}
\mathscr{T}_{i} u_{i}^{*}(t) & =\left.I^{\vartheta_{i}+\omega_{i}^{* *}} f_{i}\left(t, u_{i}^{*}(t)\right)\right|_{t=1}-I^{\vartheta_{i}+\omega_{i}^{* *}} f_{i}\left(t, u_{i}^{*}(t)\right) \\
& =\frac{1}{\Gamma\left(\vartheta_{i}+\omega_{i}^{* *}\right)} \int_{0}^{1}(1-s)^{\vartheta_{i}+\omega_{i}^{* *}-1} f_{i}\left(s, u_{i}^{*}(s)\right) \mathrm{d} s-\frac{1}{\Gamma\left(\vartheta_{i}+\omega_{i}^{* *}\right)} \int_{0}^{t}(t-s)^{\vartheta_{i}+\omega_{i}^{* *}-1} f_{i}\left(s, u_{i}^{*}(s)\right) \mathrm{d} s \\
& =\int_{0}^{1} K_{\omega_{i}, \vartheta_{i}}(t, s) f_{i}\left(s, u_{i}^{*}(s)\right) \mathrm{d} s,
\end{aligned}
$$

where $K_{\omega_{i}, 9_{i}}(s, t)$ are Green's functions defined in (3), for $i=1,2, \ldots, m$. Then, the solutions of fractional-order system (1) are the fixed points of the operator $\mathscr{T}_{i}$ defined in (8).

Also, with the help of (3), Green's functions $K_{\omega_{i}}(s, t)>0$ for the following cases. For $t \leq s$, we have $K_{\omega_{i}}(s, t)=$ $(1-s)^{\omega_{i}^{* *}+9_{i}-1}$, which is obviously positive. And for $t \geq s$, we have $K_{\omega_{i}}(s, t)=(1-s)^{\omega_{i}^{* *}+\vartheta_{i}-1}-(t-s)^{\omega_{i}^{* *}+9_{i}-1}$. Since $(1-s) \geq(t-s)$, for all $t, s \in[0,1]$, and as per our assumption $\omega_{i}^{* *}+\vartheta_{i}>1$, we have $(1-s)^{\omega_{i}^{* *}+\vartheta_{i}-1} \geq$ $(t-s)^{\omega_{i}^{* *}+\vartheta_{i}-1}$. Ultimately, Green's functions are positive.

Lemma 2. Let $f_{i} \in \mathscr{C}=\mathscr{C}\left([0,1] \times \mathscr{R}_{e}, \mathscr{R}_{e}\right)$ be continuous functions for all $i=1,2, \ldots, m$ and there exist some positive constants $\lambda_{i} \in \mathscr{R}_{e}$, for $u_{l}^{*}, u_{j}^{*} \in \mathscr{C}$ and $t \in[0, k]$, such that

$$
\left|f_{i}\left(t, u_{l}^{*}\right)-f_{i}\left(t, u_{j}^{*}\right)\right| \leq \lambda_{i}\left|u_{l}^{*}-u_{j}^{*}\right|
$$

for all $i=1,2, \ldots, m$ and $\beta_{i}=\lambda_{i}\left(1+k^{9_{i}+\omega_{i}^{* *}}\right)\left(\lambda_{i} \eta_{i}+\wp\right) /$ $\Gamma\left(\vartheta_{i}+\omega_{i}^{* *}+1\right)<1$, for $i=1,2, \ldots, m$. Then, the system of fractional order (1) has a unique solution.

Proof. Let us consider $\sup _{t \in[0, k]}\left|f_{i}(t, 0)\right|=\wp<\infty$ and $\mathcal{S}_{\eta_{i}}=\left\{u \in \mathscr{C}\left([0, k], \mathscr{R}_{e}\right):\|u\|<\eta_{i}\right\}$, where $\max _{t \in[0, k]} \lambda_{i}$, for $i=1,2, \ldots, m$ and $k \geq 1$.

For $u_{i}^{*} \in \mathcal{S}_{\eta}$, for $i=1,2, \ldots, m$ and $t \in[0, k]$, we have

$$
\begin{aligned}
\left|f_{i}\left(t, u_{i}^{*}(t)\right)\right| & =\left|f_{i}\left(t, u_{i}^{*}(t)\right)-f_{i}(t, 0)+f_{i}(t, 0)\right| \\
& \leq\left|f_{i}\left(t, u_{i}^{*}(t)\right)-f_{i}(t, 0)\right|+\left|f_{i}(t, 0)\right| \\
& \leq \lambda_{i}\left|u_{i}^{*}(t)\right|+\left|f_{i}(t, 0)\right| \\
& \leq \lambda_{i} \eta_{i}+\wp .
\end{aligned}
$$
obtain

With the help of (8) and (10), for $i=1,2, \ldots, m$, we

$$
\begin{aligned}
\left|\mathscr{T}_{i} u_{i}^{*}(t)\right| & =\left|\frac{1}{\Gamma\left(\vartheta_{i}+\omega_{i}^{* *}\right)} \int_{0}^{1}(1-s)^{\vartheta_{i}+\omega_{i}^{* *}-1} f_{i}\left(s, u_{i}^{*}(s)\right) \mathrm{d} s-\frac{1}{\Gamma\left(\vartheta_{i}+\omega_{i}^{* *}\right)} \int_{0}^{t}(t-s)^{\vartheta_{i}+\omega_{i}^{* *}-1} f_{i}\left(s, u_{i}^{*}(s)\right) \mathrm{d} s\right| \\
& \leq \frac{1}{\Gamma\left(\vartheta_{i}+\omega_{i}^{* *}\right)} \int_{0}^{1}(1-s)^{\vartheta_{i}+\omega_{i}^{* *}-1}\left|f_{i}\left(s, u_{i}^{*}(s)\right)\right| \mathrm{d} s+\frac{1}{\Gamma\left(\vartheta_{i}+\omega_{i}^{* *}\right)} \int_{0}^{t}(t-s)^{\vartheta_{i}+\omega_{i}^{* *}-1}\left|f_{i}\left(s, u_{i}^{*}(s)\right)\right| \mathrm{d} s \\
& \leq \frac{\lambda_{i} \eta_{i}+\wp}{\Gamma\left(\vartheta_{i}+\omega_{i}^{* *}\right)} \int_{0}^{1}(1-s)^{\vartheta_{i}+\omega_{i}^{* *}-1} \mathrm{~d} s+\frac{\lambda_{i} \eta_{i}+\wp}{\Gamma\left(\vartheta_{i}+\omega_{i}^{* *}\right)} \int_{0}^{t}(t-s)^{\vartheta_{i}+\omega_{i}^{* *}-1} \mathrm{~d} s \\
& =\frac{\lambda_{i} \eta_{i}+\wp}{\Gamma\left(\vartheta_{i}+\omega_{i}^{* *}+1\right)}\left(1+t^{\vartheta_{i}+\omega_{i}^{* *}}\right) \\
& \leq \frac{\left(1+k^{\vartheta_{i}+\omega_{i}^{* *}}\right)\left(\lambda_{i} \eta_{i}+\wp\right)}{\Gamma\left(\vartheta_{i}+\omega_{i}^{* *}+1\right)}
\end{aligned}
$$


This implies $\mathscr{T}_{i} \mathcal{S}_{\eta_{i}} \subset \mathcal{S}_{\eta_{i}}$. Furthermore, we assume $u_{l}^{*}, u_{j}^{*} \in \mathscr{C}\left([0, k], \mathscr{R}_{e}\right)$; for all $t \in[0, k]$, we have

$$
\begin{aligned}
\left|\mathscr{T}_{i} u_{l}^{*}(t)-\mathscr{T}_{i} u_{j}^{*}(t)\right|= & \frac{1}{\Gamma\left(\vartheta_{i}+\omega_{i}^{* *}\right)} \int_{0}^{1}(1-s)^{\vartheta_{i}+\omega_{i}^{* *}-1}\left|f_{i}\left(s, u_{l}^{*}(s)\right)-f_{i}\left(s, u_{j}^{*}(s)\right)\right| \mathrm{d} s \\
& +\frac{1}{\Gamma\left(\vartheta_{i}+\omega_{i}^{* *}\right)} \int_{0}^{t}(t-s)^{\vartheta_{i}+\omega_{i}^{* *}-1}\left|f_{i}\left(s, u_{l}^{*}(s)\right)-f\left(s, u_{j}^{*}(s)\right)\right| \mathrm{d} s \\
\leq & \frac{\lambda_{i}\left(\left|u_{l}^{*}-u_{j}^{*}\right|\right)}{\Gamma\left(\vartheta_{i}+\omega_{i}^{* *}\right)} \int_{0}^{1}(1-s)^{\vartheta_{i}+\omega_{i}^{* *}-1} \mathrm{~d} s+\frac{\lambda_{i}\left(\left|u_{l}^{*}-u_{j}^{*}\right|\right)}{\Gamma\left(\vartheta_{i}+\omega_{i}^{* *}\right)} \int_{0}^{t}(t-s)^{\vartheta_{i}+\omega_{i}^{* *}-1} \mathrm{~d} s \\
\leq & \frac{\lambda_{i}\left(1+k^{\vartheta_{i}+\omega_{i}^{* *}}\right)}{\Gamma\left(\vartheta_{i}+\omega_{i}^{* *}+1\right)}\left|u_{l}^{*}-u_{j}^{*}\right| \leq \beta_{i}\left|u_{l}^{*}-u_{j}^{*}\right|,
\end{aligned}
$$

for $i=1,2, \ldots, m$. Ultimately, the operator is a contraction and by Banach's fixed-point theorem, the system of fractional order (1) has a unique solution.

Theorem 1. Assume that the $f_{i} \in \mathscr{C}\left([0, k] \times \mathscr{R}_{e}, \mathscr{R}_{e}\right)$, for $i=1,2, \ldots, m$. Then, fractional-order system (1) has a solution provided that the assumptions of Lemma 2 are satisfied.
Proof. However, in Lemma 2, we have studied that the operator $\mathscr{T}_{i}$ is a bounded operator and $\mathscr{T}_{i} \mathcal{S}_{\eta_{i}} \subset \mathcal{S}_{\eta_{i}}$. Now, in order to prove the existence of solution of problem (1), we move towards the equicontinuity of the $\mathscr{T}_{i}$. For this, let us assume $t_{1}, t_{2} \in[0, k]$ with $t_{2}>t_{1}$, and consider

$$
\begin{aligned}
\left|\mathscr{T}_{i} u_{i}^{*}\left(t_{2}\right)-\mathscr{T}_{i} u_{i}^{*}\left(t_{1}\right)\right| & =\left|\frac{1}{\Gamma\left(\vartheta_{i}+\omega_{i}^{* *}\right)} \int_{0}^{t_{2}}\left(t_{2}-s\right)^{\vartheta_{i}+\omega_{i}^{* *}-1} f_{i}\left(s, u_{i}^{*}(s)\right) \mathrm{d} s-\frac{1}{\Gamma\left(\vartheta_{i}+\omega_{i}^{* *}\right)} \int_{0}^{t_{1}}\left(t_{1}-s\right)^{\vartheta_{i}+\omega_{i}^{* *}-1} f_{i}\left(s, u_{i}^{*}(s)\right) \mathrm{d} s\right| \\
& \leq \frac{\lambda_{i} \eta_{i}+\wp}{\Gamma\left(\vartheta_{i}+\omega_{i}^{* *}+1\right)}\left(\int_{0}^{t_{2}}\left(t_{2}-s\right)^{\vartheta_{i}+\omega_{i}^{* *}-1} \mathrm{~d} s-\int_{0}^{t_{1}}\left(t_{1}-s\right)^{\vartheta_{i}+\omega_{i}^{* *}-1} \mathrm{~d} s\right)=\frac{\lambda_{i} \eta_{i}+\wp}{\Gamma\left(\vartheta_{i}+\omega_{i}^{* *}+1\right)}\left(t_{2}^{\vartheta_{i}+\omega_{i}^{* *}}-t_{1}^{\vartheta_{i}+\omega_{i}^{* *}}\right) .
\end{aligned}
$$

This implies $\left|\mathscr{T}_{i} u_{i}^{*}\left(t_{2}\right)-\mathscr{T}_{i} u_{i}^{*}\left(t_{1}\right)\right| \longrightarrow 0$ as $t_{2} \longrightarrow t_{1}$. This implies that the operator $\mathscr{T}_{i}$ is equicontinuous. Next, we show that $\mathscr{A}=\left\{u^{*} \in \mathscr{C}\left([0, k], \mathscr{R}_{e}\right): u^{*}=\hbar \mathscr{T}_{i}\left(u^{*}\right)\right.$, for $\hbar \in[0,1]\}$ is bounded. For this, consider

$$
\begin{aligned}
\left\|u^{*}\right\|= & \left|\hbar \mathscr{T}_{i} u_{i}^{*}(t)\right|=\hbar\left|\frac{1}{\Gamma\left(\vartheta_{i}+\omega_{i}^{* *}\right)} \int_{0}^{1}(1-s)^{\vartheta_{i}+\omega_{i}^{* *}-1} f_{i}\left(s, u_{i}^{*}(s)\right) \mathrm{d} s-\frac{1}{\Gamma\left(\vartheta_{i}+\omega_{i}^{* *}\right)} \int_{0}^{t}(t-s)^{\vartheta_{i}+\omega_{i}^{* *}-1} f_{i}\left(s, u_{i}^{*}(s)\right) \mathrm{d} s\right| \\
\leq & \frac{\hbar}{\Gamma\left(\vartheta_{i}+\omega_{i}^{* *}\right)} \int_{0}^{1}(1-s)^{\vartheta_{i}+\omega_{i}^{* *}-1}\left|f_{i}\left(s, u_{i}^{*}(s)\right)-f_{i}(s, 0)+f_{i}(s, 0)\right| \mathrm{d} s \\
& +\frac{\hbar}{\Gamma\left(\vartheta_{i}+\omega_{i}^{* *}\right)} \int_{0}^{t}(t-s)^{\vartheta_{i}+\omega_{i}^{* *}-1}\left|f_{i}\left(s, u_{i}^{*}(s)\right)-f_{i}(s, 0)+f_{i}(s, 0)\right| \mathrm{d} s \\
\leq & \frac{\hbar\left(\lambda_{i}\left\|u^{*}\right\|+\wp\right)}{\Gamma\left(\vartheta_{i}+\omega_{i}^{* *}\right)} \int_{0}^{1}(1-s)^{\vartheta_{i}+\omega_{i}^{* *}-1} \mathrm{~d} s+\frac{\hbar\left(\lambda_{i}\left\|u^{*}\right\|+\wp\right)}{\Gamma\left(\vartheta_{i}+\omega_{i}^{* *}\right)} \int_{0}^{t}(t-s)^{\vartheta_{i}+\omega_{i}^{* *}-1} \mathrm{~d} s \leq \frac{\hbar\left(1+k^{\vartheta_{i}+\omega_{i}^{* *}}\right)\left(\lambda_{i}\left\|u^{*}\right\|+\wp\right)}{\Gamma\left(\vartheta_{i}+\omega_{i}^{* *}+1\right)} .
\end{aligned}
$$


By the help of (14), we have

$$
\left\|u^{*}\right\| \leq \frac{\hbar\left(1+k^{\vartheta_{i}+\omega_{i}^{* *}}\right) \wp}{\Gamma\left(\vartheta_{i}+\omega_{i}^{* *}+1\right)-\hbar\left(1+k^{\vartheta_{i}+\omega_{i}^{* *}}\right) \lambda_{i}},
$$

for $i=1,2, \ldots, m$. Hence, by (15), we have that the set $\mathscr{A}$ is bounded. Therefore, by Leray-Schauder alternative theorem, operator $\mathscr{T}_{i}$ has at least one fixed point which is the solution of fractional-order system (1).

\section{Hyers-Ulam Stability}

In this section, we are presenting the Hyers-Ulam stability analysis for hybrid fractional differential equation (8).

Definition 1. Fractional integral system (8) is said to be Hyers-Ulam stable, if there exists a constant $\zeta>0$, such that for a given $\varphi>0$ and for each solution $u_{i}^{*}$ of the inequality

$$
\left\|u_{i}^{*}-\mathscr{T}_{i} u_{i}^{*}\right\|_{1}<\varphi,
$$

there exists a solution $\overline{u_{i}^{*}}(t)$ of integral system (8),

$$
\bar{u}_{i}^{*}(t)=\mathscr{T}_{i} \bar{u}_{i}^{*}(t),
$$

such that

$$
\left\|u_{i}^{*}-\bar{u}_{i}^{*}\right\|<\varphi \zeta
$$

Theorem 2. Assume that $f_{i} \in \mathscr{C}\left(\left[[0, k] \times \mathscr{R}_{e}, \mathscr{R}_{e}\right)\right.$, for $i=1,2, \ldots, m$. Then, fractional-order system (1) is Hyers-Ulam stable provided that the assumptions of Lemma 2 are satisfied.

Proof. Let $u_{i}^{*} \in \mathscr{C}$ satisfy the inequality (16) and $\overline{u_{i}^{*}} \in \mathscr{C}$ be a solution of BVP (1) satisfying integral system (8). By the help of (16) and (17), consider the following norm:

$$
\left\|u_{i}^{*}-\bar{u}_{i}^{*}\right\|=\left\|u_{i}^{*}-\mathscr{T}_{i} u_{i}^{*}+\mathscr{T}_{i} u_{i}^{*}-\bar{u}_{i}^{*}\right\| \leq\left\|u_{i}^{*}-\mathscr{T}_{i} u_{i}^{*}\right\|+\left\|\mathscr{T}_{i} u_{i}^{*}-\mathscr{T}_{i} \bar{u}_{i}^{*}\right\| \leq \varphi_{i}+\lambda_{i}\left\|u_{i}^{*}-\bar{u}_{i}^{*}\right\|,
$$

for $i=1,2, \ldots, m$. This further implies that

$$
\left\|u_{i}^{*}-\bar{u}_{i}^{*}\right\| \leq \frac{\varphi}{1-\lambda_{i}},
$$

with $\zeta_{i}=1 / 1-\lambda_{i}$, for $i=1,2, \ldots, m$. Therefore, system ( 8$)$ is Hyers-Ulam stable which implies the stability of fractionalorder system (1).

\section{Application}

In this section, we give a specific example of fractional-order system (1) which is a fractional-order extension of the COVID-19 model given in [15]:

$$
\begin{aligned}
& \mathscr{D}_{0}^{\vartheta_{1}} \mathcal{S}=\Lambda^{*}-\frac{\alpha^{*} \mathcal{S} \mathscr{I}_{1}}{1+m \mathscr{I}_{1}^{2}}-\frac{\beta^{*} \mathcal{S} \mathscr{I}_{2}}{1+k \mathscr{I}_{2}^{2}}-\delta^{*} \mathcal{S}, \\
& \mathscr{D}_{0}^{\vartheta_{2}} \mathrm{E}_{1}=\frac{\alpha^{*} \mathcal{S} \mathscr{I}_{1}}{1+m \mathscr{I}_{1}^{2}}-\left(\gamma_{1}^{*}+\delta^{*}\right) \mathrm{E}_{1}, \\
& \mathscr{D}_{0}^{\vartheta_{3}} \mathrm{E}_{2}=\frac{\beta^{*} \mathcal{S} \mathscr{I}_{2}}{1+k \mathscr{I}_{2}^{2}}-\left(\gamma_{2}^{*}+\delta^{*}\right) \mathrm{E}_{2}, \\
& \mathscr{D}_{0}^{\vartheta_{4}} \mathscr{I}_{1}=\gamma_{1}^{*} \mathrm{E}_{1}-\left(\mu_{1}^{*}+\delta^{*}\right) \mathscr{I}_{1}, \\
& \mathscr{D}_{0}^{\vartheta_{5}} \mathscr{I}_{2}=\gamma_{2}^{*} \mathrm{E}_{2}-\left(\mu_{2}^{*}+\delta^{*}\right) \mathscr{I}_{2}, \\
& \mathscr{D}_{0}^{9_{6}} \mathscr{R}=\mu_{1}^{*} \mathscr{I}_{1}+\mu_{2}^{*} \mathscr{I}_{2}-\delta^{*} \mathscr{R} .
\end{aligned}
$$

Here, $\vartheta_{i} \in(0,1]$, for $i=1,2, \ldots, 6, \quad\left(u_{1}^{*}, u_{2}^{*}, \ldots, u_{6}^{*}\right)=$ $\left(\mathcal{S}, \mathscr{E}_{1}, \mathscr{E}_{2}, \mathscr{I}_{1}, \mathscr{I}_{2}, \mathscr{R}\right), \quad f_{1}=\Lambda^{*}-\alpha^{*} \mathcal{S} \mathscr{I}_{1} / 1+m \mathscr{I}_{1}^{2}-\beta^{*}$ $\mathcal{S} \mathscr{I}_{2} / 1+k \mathscr{J}_{2}^{2}-\delta^{*} \mathcal{S}, f_{2}=\alpha^{*} \mathcal{S} \mathscr{I}_{1} / 1+m \mathscr{I}_{1}^{2}-\left(\gamma_{1}^{*}+\delta^{*}\right) \mathscr{E}_{1}$, $f_{3}=\beta^{*} \mathcal{S} \mathscr{I}_{2} / 1+k \mathscr{I}_{2}^{2}-\left(\gamma_{2}^{*}+\delta^{*}\right) \mathscr{E}_{2}, \quad f_{4}=\gamma_{1}^{*} \mathscr{E}_{1}-\left(\mu_{1}^{*}+\right.$ $\left.\delta^{*}\right) \mathscr{I}_{1}, \quad f_{5}=\gamma_{2}^{*} \mathscr{E}_{2}-\left(\mu_{2}^{*}+\delta^{*}\right) \mathscr{I}_{2}, \quad$ and $f_{6}=\mu_{1}^{*} \mathscr{I}_{1}+\mu_{2}^{*}$ $\mathscr{I}_{2}-\delta^{*} \mathscr{R}$.

Fractional-order model (21) has six compartments. $\mathcal{S}(t)$ represents the susceptible class, $\mathscr{E}_{1}(t)$ and $\mathscr{E}_{2}(t)$ are latent individuals, $\mathscr{I}_{1}(t)$ and $\mathscr{I}_{2}(t)$ are infected individuals, and $\mathscr{R}(t)$ is the recovered class. The parameters include the following: $\Lambda$ is the recruitment rate, $1 / \delta$ is the average life of the population, $\alpha$ is the infection rate of strain $1, \beta$ is the infection rate of strain $2,1 / \mu_{1}$ is the average infection period for strain 1 , $1 / \mu_{2}$ is the average infection period for strain $2,1 / \gamma_{1}$ is the average latency rate for strain $1,1 / \gamma_{2}$ is the average latency rate for strain $2, m$ is the psychological effect of strain 1 , and $k$ is the psychological effect of strain 2 .

The existence of solution of (21) is ensured by Theorem 1. The stability of (21) is also ensured by Theorem 2 . Now, we give the numerical scheme for the simulation of (21) as follows. By applying the fractional-order Riemann-Liouville integral operator for the equispace intervals of $[0, k]$, we get the following form: 


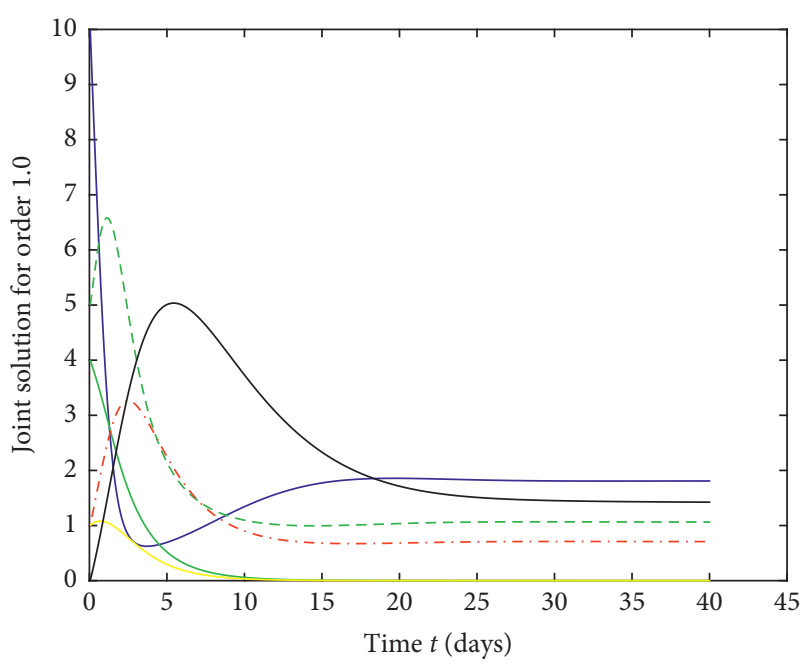

$-S$

Figure 1: Joint solution of (21) for order 1.0.

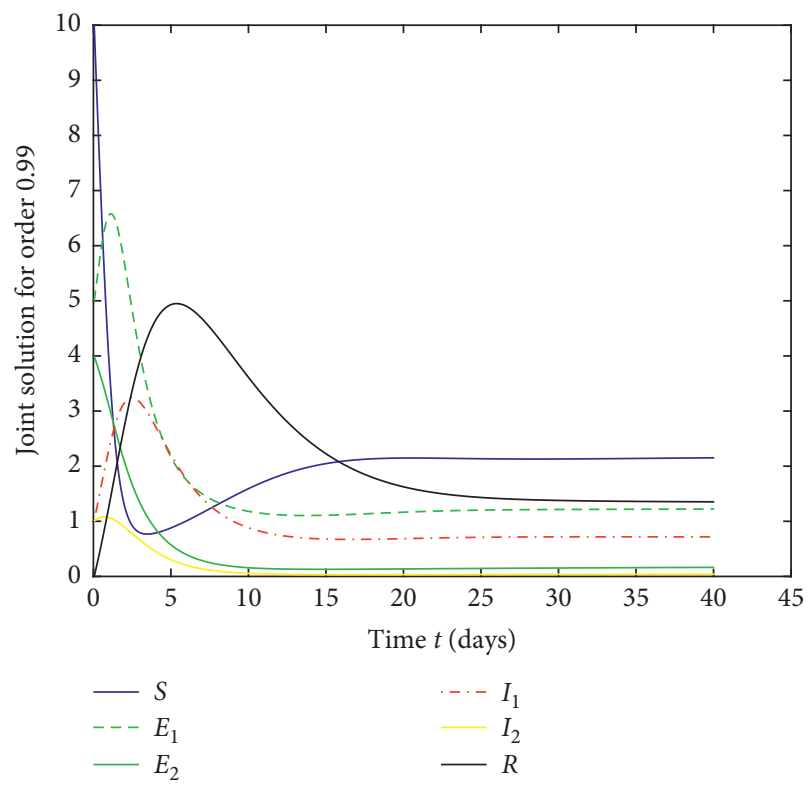

FIGURE 2: Joint solution of (21) for order 0.99.

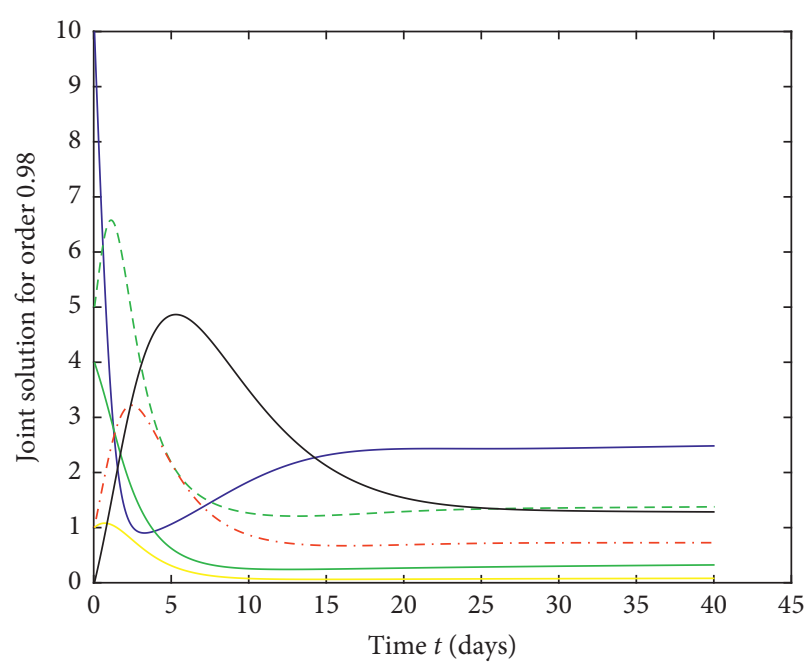

FIGURE 3: Joint solution of (21) for order 0.98.

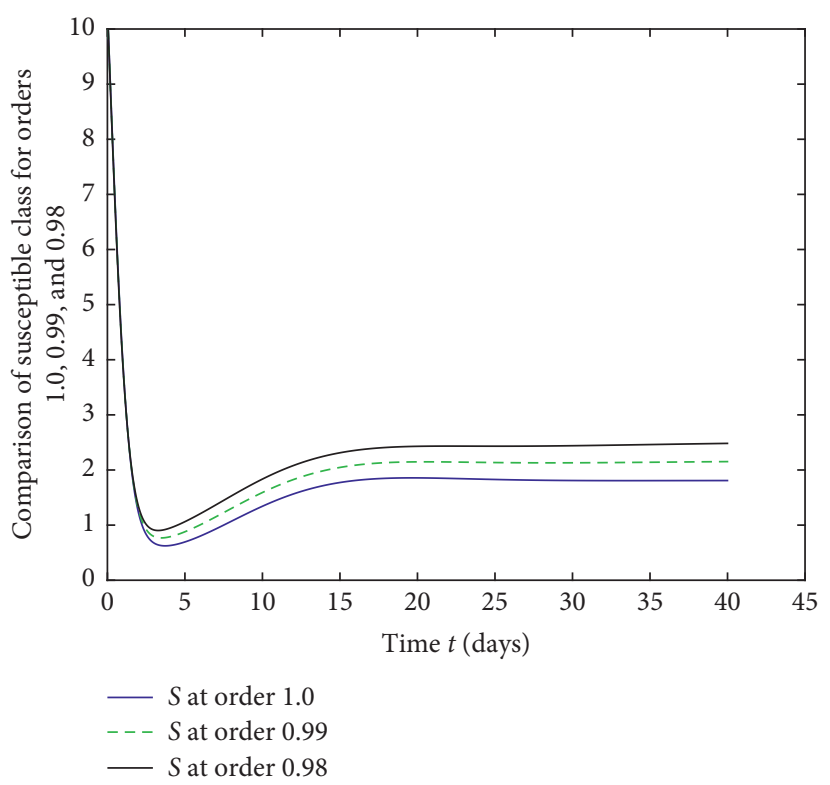

Figure 4: Comparison of $S(t)$ for orders 1.0, 0.99, and 0.98 . 


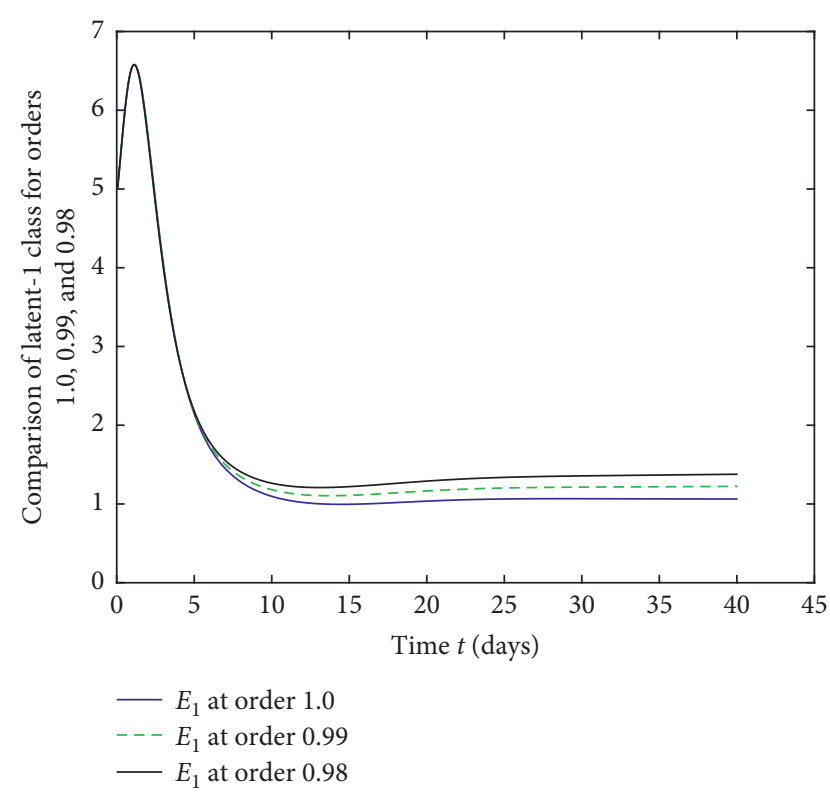

FIgURE 5: Comparison of $E_{1}(t)$ for orders 1.0, 0.99, and 0.98 .

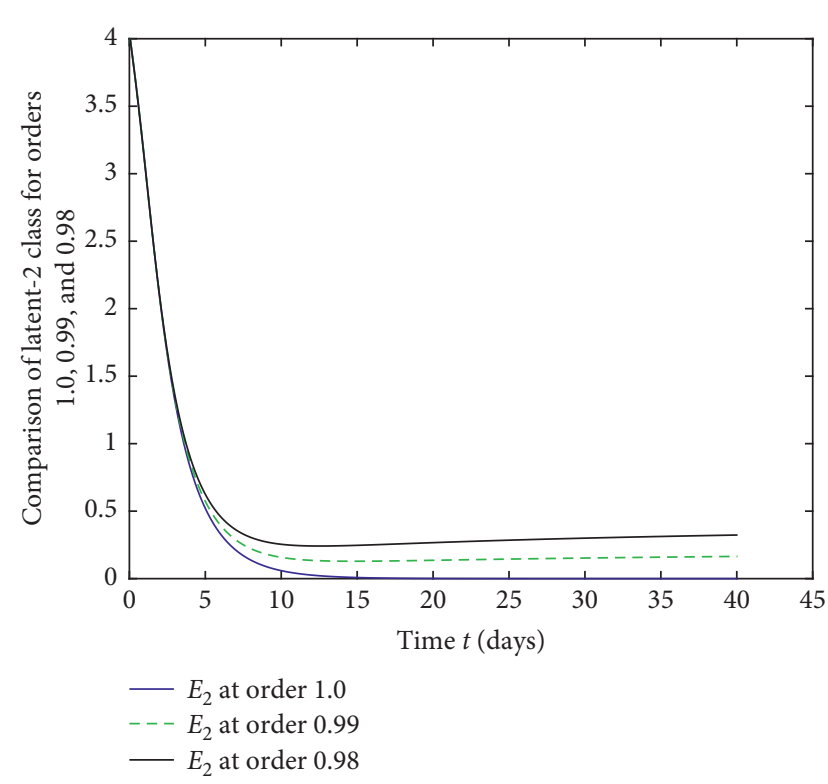

Figure 6: Comparison of $I_{1}(t)$ for orders 1.0, 0.99, and 0.98 .

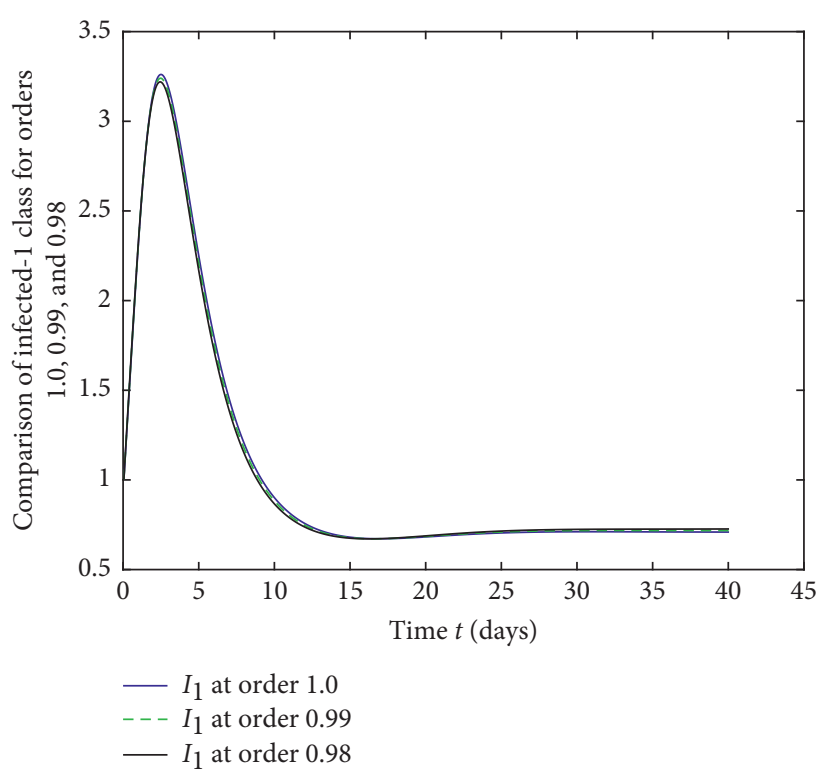

Figure 7: Comparison of $E_{2}(t)$ for orders 1.0, 0.99, and 0.98.

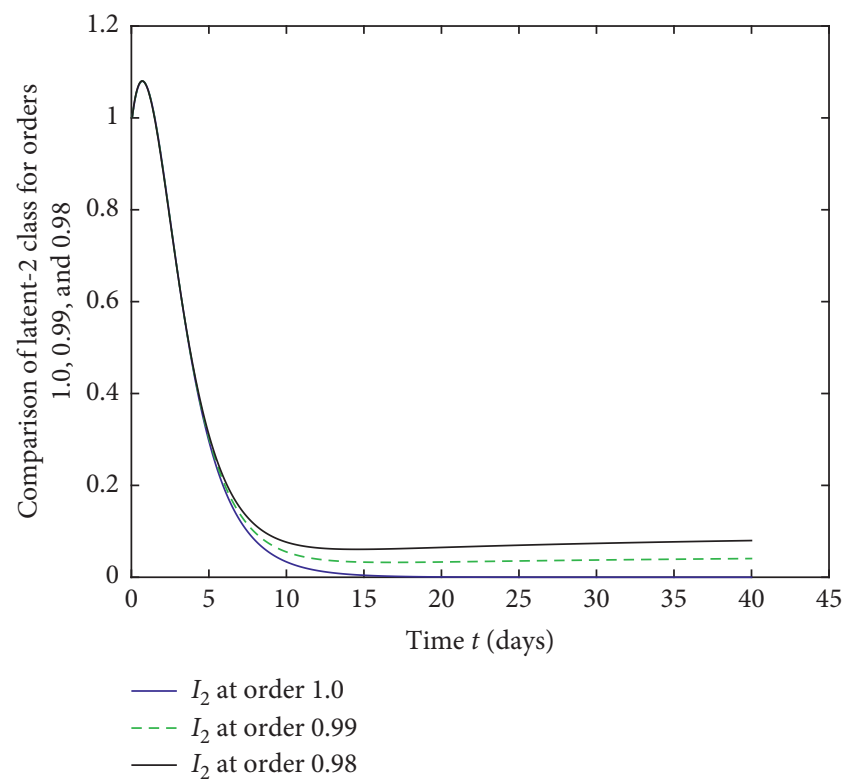

Figure 8: Comparison of $R(t)$ for orders 1.0, 0.99, and 0.98 . 


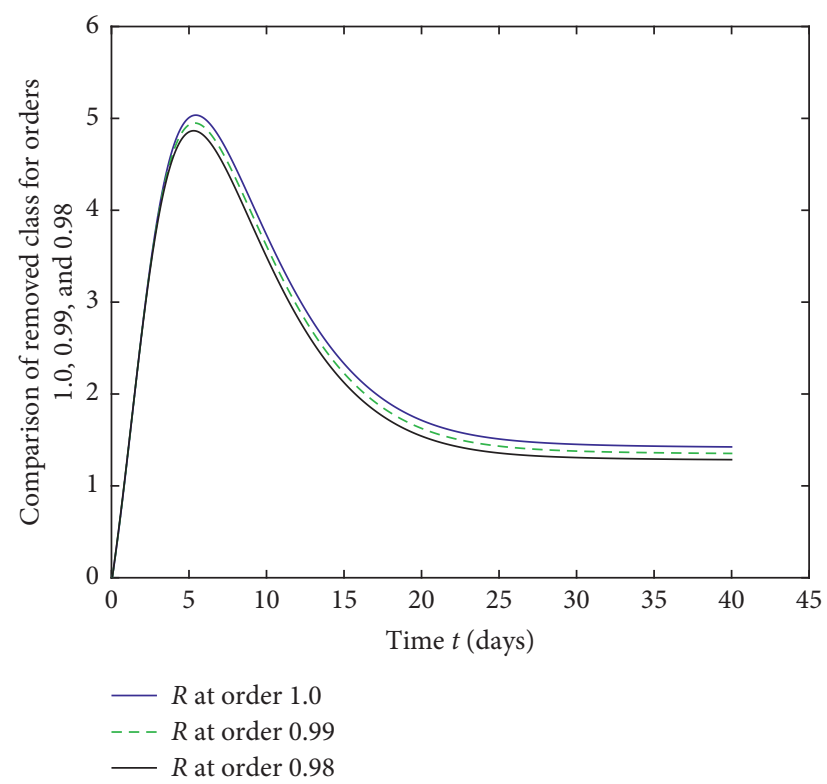

Figure 9: Comparison of $I_{2}(t)$ for orders 1.0, 0.99, and 0.98.

$$
\begin{aligned}
& \mathcal{S}_{n+1}=\mathcal{S}_{0}+\frac{h^{\vartheta_{1}}}{\Gamma\left(\mathcal{\vartheta}_{1}+1\right)} \times \sum_{k=0}^{n}\left((n-k+1)^{\vartheta_{1}}-(n-k)^{\vartheta_{1}}\right)\left(\Lambda^{*}-\frac{\alpha^{*} \mathcal{S}_{n} \mathcal{I}_{1_{n}}}{1+m \mathcal{F}_{1_{n}}^{2}}-\frac{\beta^{*} \mathcal{S}_{n} \mathcal{F}_{2_{n}}}{1+k \mathcal{F}_{2_{n}}^{2}}-\delta^{*} \mathcal{S}_{n}\right), \\
& \varepsilon_{1_{n}}=\varepsilon_{0}+\frac{h^{\vartheta_{2}}}{\Gamma\left(\vartheta_{2}+1\right)} \times \sum_{k=0}^{n}\left((n-k+1)^{\vartheta_{2}}-(n-k)^{\vartheta_{2}}\right)\left(\frac{\alpha^{*} \mathcal{S}_{n} \mathcal{I}_{1_{n}}}{1+m \mathcal{F}_{1_{n}}^{2}}-\left(\gamma_{1}^{*}+\delta^{*}\right) \mathrm{E}_{1_{n}}\right), \\
& \varepsilon_{2_{n}}=\varepsilon_{2_{0}}+\frac{h^{\vartheta_{3}}}{\Gamma\left(\vartheta_{3}+1\right)} \times \sum_{k=0}^{n}\left((n-k+1)^{\vartheta_{3}}-(n-k)^{\vartheta_{3}}\right)\left(\frac{\beta^{*} \mathcal{S}_{n} \mathcal{I}_{2_{n}}}{1+k \mathcal{F}_{2_{n}}^{2}}-\left(\gamma_{2}^{*}+\delta^{*}\right) \mathrm{E}_{2_{n}}\right) \text {, } \\
& \mathscr{I}_{1_{n}}=\mathscr{I}_{1_{0}}+\frac{h^{\vartheta_{4}}}{\Gamma\left(\vartheta_{4}+1\right)} \times \sum_{k=0}^{n}\left((n-k+1)^{\vartheta_{4}}-(n-k)^{\vartheta_{4}}\right)\left(\gamma_{1}^{*} \mathrm{E}_{1_{n}}-\left(\mu_{1}^{*}+\delta^{*}\right) \mathscr{I}_{1_{n}}\right), \\
& \mathscr{I}_{2_{n}}=\mathscr{I}_{2_{0}}+\frac{h^{\vartheta_{5}}}{\Gamma\left(\vartheta_{5}+1\right)} \times \sum_{k=0}^{n}\left((n-k+1)^{\vartheta_{5}}-(n-k)^{\vartheta_{5}}\right)\left(\gamma_{2}^{*} \mathrm{E}_{2_{n}}-\left(\mu_{2}^{*}+\delta^{*}\right) \mathscr{I}_{2_{n}}\right), \\
& \mathscr{R}_{n}=\mathscr{R}_{0}+\frac{h^{\vartheta_{6}}}{\Gamma\left(\vartheta_{6}+1\right)} \times \sum_{k=0}^{n}\left((n-k+1)^{\vartheta_{6}}-(n-k)^{\vartheta_{6}}\right)\left(\mu_{1}^{*} \mathscr{I}_{1_{n}}+\mu_{2}^{*} \mathscr{I}_{2_{n}}-\delta^{*} \mathscr{R}_{n}\right) .
\end{aligned}
$$

\section{Computational Results}

Here, we test the numerical scheme given in (22) for the numerical results of fractional-order COVID-19 model (21), considering the parametric values, and $\vartheta_{i}=$ $1.0,0.99$, and 0.98 , for $i=1,2, \ldots, 6$, and the initial values $\mathcal{S}(0)=10, \mathscr{E}_{1}(0)=5, \mathscr{E}_{2}(0)=4, \mathscr{I}_{1}(0)=1, \mathscr{I}_{2}(0)=1$, and $\mathscr{R}(0)=0$.

In Figure 1, we have given the numerical solution of COVID-19 model (21) for the order 1.0. Also, Figures 2 and 3 are the solutions for the fractional orders 0.99 and 0.98 , respectively. These graphs show that the fractional-order solutions of model (21) are similar in behavior as to the solution of the classical model of the order 1.0. This shows the accuracy of our scheme given in (22).

In Figure 4, we have given a comparative study of the susceptible class which has a decrease in the early 5 days and later on a slight increase and then a stability is observed for the orders 1.0,0.99, and 0.98. In Figure 5, we have given a comparative study of the $E_{1}(t)$ for the orders $1.0,0.99$, and 0.98 . We have observed that the behavior of the fractional-order results are similar to the classical integer order and in this class, there is a slight increase and then there is a rapid decrease up to 10 days. This decrease is converted into the infected class, and we observe a rapid increase in the $I_{1}(t)$ class and then 
there is a gradual decrease of up to 14 days as given in Figure 6.

In Figure 7, we have given a comparative study of the $E_{2}(t)$ for the orders $1.0,0.99$, and 0.98 . We have observed that the behavior of the fractional-order results are similar to the classical integer order and in this class, there is a rapid decrease of up to 10 days. This decrease is converted into the infected class, and we observed a rapid increase in the $R(t)$ class and then there is a gradual decrease of up to 20 days as given in Figure 8. In Figure 9, we have presented a computational analysis of $\mathscr{I}_{2}$ class.

All the numerical computations are for the comparative study of COVID-19 model (21) and we have noticed the behavior of the joint solution as well as the individual comparison of the compartments for the orders 1.0, 0.99, and 0.98.

\section{Conclusion}

In this article, we have considered a very important class of fractional-order system of sequential differential equations (1), for the existence and stability results based on the classical fixed-point approach, and have observed that under certain necessary assumptions, suggested problem (1) has a unique solution as well as Hyers-Ulam stability. Such problems are widely applicable in the real-world situations. In the example section, we have given a COVID-19 model as a particular case of system (1). The existence of solution of (21) is ensured by Theorem 1. The Hyers-Ulam stability of (21) is guaranteed by Theorem 2. For the numerical solution of example (21), we obtained numerical scheme (22) and the scheme was tested with the real data given in the literature [15]. All the computational results ensured that the numerical simulations for fractional-order system (21) are of the same behavior as to the classical case for the order 1 .

\section{Data Availability}

The data used to support the findings of this study are included within the article.

\section{Conflicts of Interest}

The authors declare no conflicts of interest regarding the publication of this paper.

\section{Authors' Contributions}

All the authors have equal contributions in this article.

\section{Acknowledgments}

This research was funded by the Deanship of Scientific Research at Princess Nourah Bint Abdulrahman University through the Fast-Track Research Funding Program to support publication in the top journal (Grant no. 42-FTTJ-64).

\section{References}

[1] A. Atangana and S. I. Araz, "Mathematical model of COVID19 spread in Turkey and South Africa: theory, methods and applications," Advances in Difference Equations, vol. 2020, p. $658,2020$.

[2] A. Atangana and S. I. Araz, "Nonlinear equations with global differential and integral operators: existence, uniqueness with application to epidemiology," Results in Physics, vol. 20, Article ID 103593, 2020.

[3] S. Ahmad, A. Ullah, Q. M. Al-Mdallal, H. Khan, K. Shah, and A. Khan, "Fractional order mathematical modeling of COVID-19 transmission. Chaos," Solitons \& Fractals, vol. 139, Article ID 110256, 2020.

[4] A. Babakhani and V. Daftardar-Gejji, "Existence of positive solutions of nonlinear fractional differential equations," Journal of Mathematical Analysis and Applications, vol. 278, no. 2, pp. 434-442, 2003.

[5] N. H. Tuan, H. Mohammadi, and S. Rezapour, "A mathematical model for COVID-19 transmission by using the Caputo fractional derivative," Chaos, Solitons \& Fractals, vol. 140, Article ID 110107, 2020.

[6] Z. A. Khan, "Hadamard type fractional differential equations for the system of integral inequalities on time scales," Integral Transforms and Special Functions, vol. 31, no. 5, pp. 412-423, 2020.

[7] Z. A. Khan, K. Shah, A. Khan, and H. Khan, "Nonlinear discrete fractional sum inequalities related to the theory of discrete fractional calculus with applications," Journal of Function Spaces, vol. 2021, Article ID 8734535, 8 pages, 2021.

[8] K. Deimling, Nonlinear Functional Analysis, Springer-Verlag, New York, NY, USA, 1985.

[9] P. Samui, J. Mondal, and S. Khajanchi, "A mathematical model for COVID-19 transmission dynamics with a case study of India," Chaos, Solitons, and Fractals, vol. 140, Article ID 110173, 2020.

[10] S. Zhang, "The existence of a positive solution for a nonlinear fractional differential equation," Journal of Mathematical Analysis and Applications, vol. 252, no. 2, pp. 804-812, 2000.

[11] D. Okuonghae and A. Omame, "Analysis of a mathematical model for COVID-19 population dynamics in Lagos, Nigeria," Chaos, Solitons, and Fractals, vol. 139, Article ID $110032,2020$.

[12] B. C. Dhage, "Quadratic perturbations of periodic boundary value problems of second order ordinary differential equations," Differential Equations \& Applications, vol. 2, no. 4, pp. 465-486, 2010.

[13] B. Dhage, "Periodic boundary value problems of first order Caratheodory and discontinuous differential equations," Nonlinear Functional Analysis and Applications, vol. 13, no. 2, pp. 323-352, 2008.

[14] B. Dhage, "Basic results in the theory of hybrid differential equations with mixed perturbations of second type," Functional Differential Equations, vol. 19, pp. 1-20, 2012.

[15] A. Meskaf, O. Khyar, J. Danane, and K. Allali, "Global stability analysis of a two-strain epidemic model with non-monotone incidence rates," Chaos, Solitons \& Fractals, vol. 133, Article ID 109647, 2020. 